\title{
Positive predictive values for self-reported fractures in an adult Japanese population
}

\author{
Kazutoshi Nakamura $\cdot$ Manami Inoue • \\ Yoshikazu Kaneko $\cdot$ Shoichiro Tsugane
}

Received: 29 April 2010/Accepted: 10 July 2010/Published online: 10 August 2010

(C) The Japanese Society for Hygiene 2010

\begin{abstract}
Objectives Self-reporting provides useful information for assessing the risk factors of osteoporotic fractures in large cohort studies. However, to data, no studies in Japan have confirmed the accuracy of this approach in this context. The aim of the study reported here was to determine the positive predictive value (PPV) for the self-reporting of fractures.

Methods A total of 133 participants of the Oguni cohort in the Japan Public Health Center-based Prospective Study who reported a vertebral, upper limb, or hip fracture on the 15 -year follow-up questionnaire survey were evaluated. The accuracy of fractures was confirmed by cross-referencing medical records.

Results The average age of the participants was 72.4 (standard deviation 7.9) years. The PPV for vertebral
\end{abstract}

For the Japan Public Health Center-based Prospective Study Group.

K. Nakamura $(\square)$

Department of Community Preventive Medicine, Niigata

University Graduate School of Medical and Dental Sciences,

1-757 Asahimachi-dori, Chuo-ku, Niigata 951-8510, Japan

e-mail: kazun@med.niigata-u.ac.jp

M. Inoue $\cdot$ S. Tsugane

Epidemiology and Prevention Division,

Research Center for Cancer Prevention and Screening,

National Cancer Center, 5-1-1 Tsukiji, Chuo-ku,

Tokyo 104-0045, Japan

e-mail: stsugane@ncc.go.jp

Y. Kaneko

Oguni Clinic, 88 Oguni-machi Narasawa,

Nagaoka 949-5331, Japan fracture in the last 15 years was $17 / 20(85.0 \%)$ for women and $2 / 9(22.2 \%)$ for men, for a total of $19 / 29$ (65.5\%). PPVs for upper limb and hip fractures were as low as 30/68 (44.1\%) and 12/22 (54.5\%), respectively.

Conclusion Female self-reporting provided PPVs suitable for symptomatic vertebral fracture over 15 years and can be used as an outcome measure in large cohort studies in Japan.

Keywords Cohort studies · Fractures · Osteoporosis · Predictive value of tests

\section{Introduction}

Fractures due to osteoporosis are a growing public health concern in Japan. Osteoporotic fractures, the number of which have increased over the last two decades [1], have an adverse effect not only on patients' quality of life but also on medical care costs. Consequently, the prevention of osteoporotic fracture is of high priority. Epidemiologic studies on osteoporosis using incident fracture as an outcome are highly important in this context; however, few studies have explored the risk factors for osteoporotic fractures in Japan.

The Japan Public Health Center-based Prospective Study (JPHC Study) [2] is one of the largest population-based cohort studies in Japan, examining multiple outcomes of lifestyle-related diseases. One outcome evaluated in the JPHC Study is self-reported data on fractures. Such data may be used to analyze fracture risk factors if accurate and if they provide an appropriate positive predictive value (PPV). The aim of this study was, therefore, to determine PPVs for self-reported fractures of vertebra, upper limbs, and hip in a cohort of the JPHC Study. 


\section{Subjects and methods}

The study cohort consisted of all individuals in the Oguni cohort of the JPHC Study who reported any fracture in the 15-year follow-up questionnaire survey. Of the 2,248 respondents, 165 reported a fracture. Of these, 133 (80.6\%) participated in our study after providing written informed consent. The study protocol was approved by the Institutional Review Board of the National Cancer Center, Tokyo, Japan. A detailed description of the study design, methodology, and participant profiles of the JPHC Study has been published previously [2].

In the 15-year follow-up questionnaire, subjects were to self-report any occurrence of fractures in the lumbar region (herein defined as a vertebral fracture), upper limbs, or hip. The question was posed as: "Has your doctor(s) told you that you have a fracture in the lumbar region, upper limbs, or hip? If so, was the fracture first diagnosed in the past 5 years, 5-15 years, or earlier?" Subjects were asked not to report fractures due to high-energy trauma, including traffic or occupational accidents. We tabulated all individuals who reported any incident fracture of the vertebra, upper limbs, or hip during the 15-year follow-up period and mailed them a second questionnaire to confirm the reported fractures. The second questionnaire asked subjects to provide data on the fracture site, date of occurrence, and medical facility for treatment of the reported fracture. The accuracy of information on incident fractures was confirmed by crossreferencing medical records at the designated medical facilities. When a subject's medical record was considered discarded, the reported fracture was classified as unconfirmed. In this study, vertebral fractures included compression fractures at the lumbar and thoracic regions, which were diagnosed using criteria from the Japanese Society of Bone and Mineral Research [3], fractures of upper limbs, which included fractures of the radius, ulna, and humerus, and hip fractures, which included fractures at the femoral neck and trochanteric region. Using the above information, we calculated a PPV, i.e., the probability that a person who self-reports a fracture has a true fracture, for fractures of the vertebra, upper limbs, and hip, respectively, stratified by gender and fracture date according to the following formula: (true positives)/(true positives + false positives), where true positives are those who self-reported a fracture and had a fracture, and false positives are those who selfreported a fracture but did not have a fracture.

\section{Results}

The average age of the 133 subjects ( 87 women and 46 men) was 72.4 (standard deviation 7.9) years. Cumulatively, they reported 34 vertebral fractures, 88 upper limb fractures, and 23 hip fractures in the 15-year follow-up survey. Of these, five vertebral fractures (14.7\%), 20 upper limb fractures $(22.7 \%)$, and one hip fracture $(4.3 \%)$ could not be confirmed due to the unavailability of medical records. PPVs for self-reported fractures stratified by gender and fracture date are shown in Table 1. High PPVs were found for vertebral fracture in women, namely, $>80 \%$ in any follow-up period, whereas PPVs in men were unsatisfactorily low. PPVs for upper limb fracture were generally low, except for those occurring in women over the last 5 years. PPVs for hip fractures occurring in the most recent 5 years were higher than those occurring earlier.
Table 1 Positive predictive values (PPVs) for self-reported fractures of the vertebra, upper limbs, and hip by gender and fracture date
Values are given as a percentage, with the no. of subjects with true fracture/no. of subjects with self-reported fracture given in square parenthesis

\begin{tabular}{llll}
\hline Fracture location & \multicolumn{2}{l}{ PPV $(\%)$} & \\
\cline { 2 - 4 } & $\begin{array}{l}15-5 \text { years ago } \\
(1993-2002)\end{array}$ & $\begin{array}{l}\text { Last 5 years } \\
(2003-2008)\end{array}$ & $\begin{array}{l}\text { Total (Last 15 years) } \\
(1993-2008)\end{array}$ \\
\hline Vertebral fracture & & & \\
Women & $81.8[9 / 11]$ & $88.9[8 / 9]$ & $85.0[17 / 20]$ \\
Men & $0[0 / 5]$ & $50.0[2 / 4]$ & $22.2[2 / 9]$ \\
Total & $56.3[9 / 16]$ & $76.9[10 / 13]$ & $65.5[19 / 29]$ \\
Upper limb fracture & & & \\
Women & $39.1[9 / 23]$ & $70.8[17 / 24]$ & $55.3[26 / 47]$ \\
Men & $11.8[2 / 17]$ & $50.0[2 / 4]$ & $19.0[4 / 21]$ \\
Total & $27.5[11 / 40]$ & $67.9[19 / 28]$ & $44.1[30 / 68]$ \\
Hip fracture & & $66.7[6 / 9]$ & $60.0[9 / 15]$ \\
Women & $50.0[3 / 6]$ & $100[2 / 2]$ & $42.9[3 / 7]$ \\
Men & $20.0[1 / 5]$ & $72.7[8 / 11]$ & $54.5[12 / 22]$ \\
Total & $36.4[4 / 11]$ & &
\end{tabular}




\section{Discussion}

This is the first study to confirm the adequacy of PPV for self-reported vertebral fracture in Japanese women. Several validation studies have confirmed self-reported non-vertebral fractures in Caucasian populations [4-7]; however, very few studies have addressed the accuracy of selfreported vertebral fractures. One reason may be that vertebral fractures are not common in such populations. In contrast, vertebral fractures have been reported to be more common in Japan than in Western countries [8]. We believed that both patients and orthopedists in Japan are more likely to be attentive to this type of fracture, especially among women. It should be noted that self-reported vertebral fractures are likely to be symptomatic; thus, the results of our study cannot be applied to asymptomatic vertebral fractures.

Although a number of studies have shown the selfreporting of limb fractures to be accurate [4-7], we were unable to demonstrate accurate reporting of upper limb or hip fractures, even for those occurring in the last 5 years. We further investigated nine false positive cases of upper limb fracture reported in the last 5 years and found that five were due to high-energy trauma, one was a fracture at a different anatomical site (the clavicle), one involved fractures predating the study period, and two cases did not involve fractures. Fracture secondary to high-energy trauma primarily skewed the PPV for recent upper limb fractures. As such, data on selfreported fractures, especially for those of limbs, should be used with care when conducting a questionnaire survey, and a strategy to exclude fractures arising from high-energy trauma should be considered. An insufficient number of self-reported hip fracture cases were obtained, which should be further investigated in future studies.

A limitation of this study was that the sensitivity could not be evaluated; that is, an unknown number of cases with a fracture may not have been reported in our cohort. If the sensitivity is insufficiently high, self-reported fractures may not represent the total number of fractures occurring in a study, and a positive association may be underestimated due to a reduction of effect size. However, the sensitivity of self-reported fractures is relatively high because, unlike cancer, fractures can be readily recognized by the individual. In fact, previous studies have demonstrated sensitivities of self-reporting as high as 78 [5] and $86 \%$ [7] for all types of fragility fracture. The sensitivity of self-reported vertebral fractures has not yet been reported. Notably, self-reported vertebral fractures are very likely to be symptomatic. Indeed, in our study, all vertebral fractures confirmed in medical records were symptomatic. Thus, the accuracy of self-reported vertebral fractures should be discussed with respect to symptomatic vertebral fractures. The sensitivity of selfreporting symptomatic vertebral fractures is considered to be acceptably high, similar to that of other fragility fractures. However, the prevalence of asymptomatic vertebral fractures is estimated to be tenfold higher than that of symptomatic fractures [9]. Thus, the sensitivity of selfreporting all vertebral fractures, including symptomatic and asymptomatic fractures, must be low. Although the detection of false negative cases of self-reported fractures occurring in the distant past is difficult, the sensitivity of self-reported fractures should be explored in a future study.

In conclusion, female self-reporting provided PPVs suitable for symptomatic vertebral fracture over the 15year follow-up period and can be used as an outcome measure in large cohort studies. PPVs for other fracture types, such as hip fractures, should be further investigated with a larger sample size.

Acknowledgments We thank the following institutions for providing us with relevant medical information: Imura Orthopedic Clinic, Kobayashi Orthopedic Clinic, Nagaoka Chuo General Hospital, Nagaoka Red Cross Hospital, Nemoto Orthopedic Clinic, Oguni Clinic, Ojiya General Hospital, Tachikawa General Hospital, Tokamachi Hospital, Uonuma Hospital, and Yoshida Hospital. This study was supported by a Grant-in-Aid for Cancer Research and for the Third Term Comprehensive Control Research for Cancer from the Ministry of Health, Labour and Welfare of Japan. The authors have no conflicts of interest to report.

\section{References}

1. Morita Y, Endo N, Iga T, Tokunaga K, Ohkawa Y. The incidence of cervical and trochanteric fractures of the proximal femur in 1999 in Niigata Prefecture, Japan. J Bone Miner Metab. 2002; 20:311-8.

2. Tsugane S, Sobue T. Baseline survey of JPHC study: design and participation rate. J Epidemiol. 2001;11[Suppl 6]:S24-9.

3. Orimo H, Sugioka Y, Fukunaga M, Muto Y, Hotokebuchi T, Gorai I, Nakamura T, Kushida K, Tanaka H, Ikai T, Oh-hashi Y. Diagnostic criteria of primary osteoporosis. J Bone Mineral Metab. 1998;16:139-50.

4. Nevitt MC, Cummings SR, Browner WS, Seeley DG, Cauley JA, Vogt TM, Black DM. The accuracy of self-report of fractures in elderly women: evidence from a prospective study. Am J Epidemiol. 1992;135:490-9.

5. Honkanen K, Honkanen R, Heikkinen L, Kröger H, Saarikoski S. Validity of self-reports of fractures in perimenopausal women. Am J Epidemiol. 1999;150:511-6.

6. Ismail AA, O'Neill TW, Cockerill W, Finn JD, Cannata JB, Hoszowski K, Johnell O, Matthis C, Raspe H, Raspe A, Reeve J, Silman AJ. Validity of self-report of fractures: results from a prospective study in men and women across Europe. EPOS Study Group. European Prospective Osteoporosis Study Group. Osteoporos Int. 2000;11:248-54. 
7. Ivers RQ, Cumming RG, Mitchell P, Peduto AJ. The accuracy of self-reported fractures in older people. $\mathrm{J}$ Clin Epidemiol. 2002;55:452-7.

8. Ross PD, Fujiwara S, Huang C, Davis JW, Epstein RS, Wasnich $\mathrm{RD}$, Kodama K, Melton LJIII. Vertebral fracture prevalence in women in Hiroshima compared to Caucasians or Japanese in the US. Int J Epidemiol. 1995;24:1171-7.
9. Nakamura K, Kurahashi N, Ishihara J, Inoue M, Tsugane S, for the Japan Public Health Centre-based Prospective Study Group. Calcium intake and the 10-year incidence of self-reported vertebral fractures in women and men: the Japan Public Health Centre-based Prospective Study. Br J Nutr. 2009;101:285-94. 